\title{
The FARCOS project. First characterization of CsI(TI) crystals of the FARCOS array using charged particle beams at LNS
}

L. Quattrocchi ${ }^{1,2 a}$, L. Acosta ${ }^{5,7}$, E. De Filippo ${ }^{4}$, T. Minniti ${ }^{1,2}$, E. V. Pagano ${ }^{3,5}$, P. Russotto ${ }^{4}$,

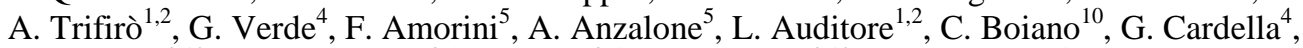

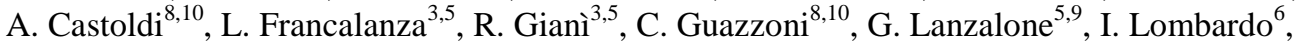

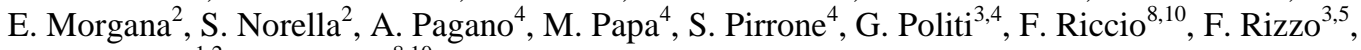
M. Trimarchi ${ }^{1,2}$, P. Zambon ${ }^{8,10}$

${ }^{1}$ INFN, Gruppo Collegato di Messina, Messina, Italy

${ }^{2}$ Università Messina, Dipartimento di Fisica e Scienze della Terra, Messina, Italy

${ }^{3}$ Università di Catania , Dipartimento di Fisica e Astronomia, Catania, Italy

${ }^{4}$ INFN, Sezione di Catania, Catania, Italy

${ }^{5}$ INFN, Laboratori Nazionali del Sud, Catania, Italy

${ }^{6}$ Università di Napoli Federico II, Dipartimento di Fisica, \& INFN sez. di Napoli, Napoli, Italy

${ }^{7}$ Departamento de Física Aplicada, Universidad de Huelva, Huelva, Spain

${ }^{8}$ Politecnico di Milano, Dipartimento di Elettronica, Informazione e Bioingegneria, Milano, Italy

${ }^{9}$ Università Kore, Enna, Italy

${ }^{10}$ INFN, Sezione di Milano, Milano, Italy

\begin{abstract}
The construction of a new array to study femtoscopy and multi-particle correlations in heavy ion collisions at intermediate energies $(\mathrm{E}=20-1000 \mathrm{AMeV})$ has been started at the INFN. The project, named FARCOS (Femtoscope ARray for COrrelations and Spectroscopy) is aimed at developing of a detection system with high pixelation capabilities in order to perform precision measurements of particle correlations for nuclear dynamics and spectroscopy. We present first detection simulations for FARCOS and first results related to the commissioning of $\mathrm{CsI}(\mathrm{Tl})$ crystals, an important detection stage of each telescope.
\end{abstract}

FARCOS has been conceived as a compact high resolution array and it will address the study of two and multi-particles correlations for nuclear dynamics and spectroscopy. The correlation between different particles emitted during a collision provide important information about space-time properties of emitting sources produced in heavy ion collisions and quantitative understanding of reaction dynamics [1]. The studies of correlation functions can also be used as a powerful tool to explore certain spectroscopic properties of unbounded states of exotic nuclei [2]. The basic telescope of FARCOS consists of two double-sided silicon strip detectors (DSSSD) 300 $\mu \mathrm{m}$ - and 1500 $\mu \mathrm{m}$-thick, as first and second stage respectively, followed by $4 \mathrm{CsI}(\mathrm{Tl})$ crystals arranged in $2 \times 2$ configuration, as a third stage. Each DSSSD features 32 horizontal and 32 vertical strips, providing 1024 equivalent

a Corresponding author: lucia.quattrocchi@unime.it

This is an Open Access article distributed under the terms of the Creative Commons Attribution License 2.0, which permits unrestricted use, distribution, and reproduction in any medium, provided the original work is properly cited. 
pixel of $2 \times 2 \mathrm{~mm}^{2}$. High energy resolution combined with high angular resolution provide a good measurement of relative energy, momentum vector and hence their correlation. FARCOS has a wide dynamic range that goes from $\mathrm{MeV}$ to $\mathrm{GeV}$, since low identification threshold can be attained with pulse-shape techniques. Silicon nTD solutions are also under consideration to improve pulse-shape capabilities, as observed in some experiments [5]. Furthermore the flexible geometry allows arranging FARCOS telescopes to cope with the physical case under investigation. It has been conceived as a portable device so that it can be coupled with other existing detector such as $4 \pi$ arrays, magnetic spectrometers or neutron detectors.
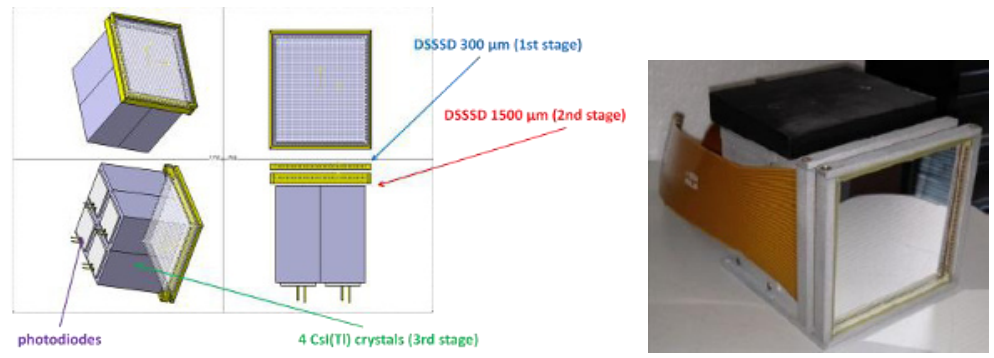

Figure 1. Left panel: schematic representation of a FARCOS telescope seen from different perspectives. Right panel: photo of one of the first prototypes of FARCOS telescope.

\section{The importance of angular resolution}

In order to understand the influence of angular resolution on reconstruction of correlation functions, preliminary simulations were carried out. It was chosen to simulate the decay of ${ }^{6} \mathrm{Li}$ in a deuteron and an alpha particle because this is one of the typical systems produced in heavy ion collisions at intermediate energies. The theoretical data were processed through a filter that simulate the event detection for every geometrical configuration of FARCOS array.
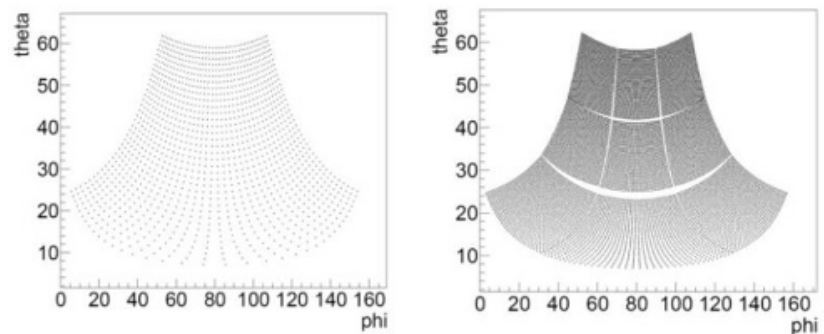

Figure 2. Angular resolution obtained with one (left panel) and nine (right panel) FARCOS telescopes covering the same angular region; the points in this plot represent the spherical coordinates of the center of each pixel.
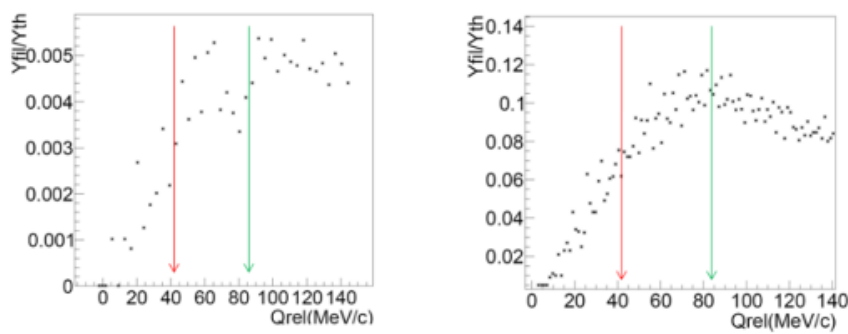

Figure 3. Scatter plots of the ratio of the number of detected particle pairs and the number of produced pairs vs. the relative momentum $\mathrm{Q}_{\mathrm{rel}}$ (left panel: one telescope; right panel: nine telescopes). 
This filter is also very useful for comparing theoretical predictions with experimental observations. Fig. 2 shows the geometrical configuration of two simulated setups that consist respectively of a single telescope and of 9 telescopes arranged in a $3 \times 3$ configuration. The two setups cover the same angular region but they have different angular resolution, as evidenced by plots in fig.2: in the first case the average polar resolution is about $1.4^{\circ}$, while in the second one it is $0.5^{\circ}$. The scatter plots shown in fig. 3 report the ratio between number of pairs detected by simulated configurations and the total number produced as a function of relative momentum $\mathrm{Q}_{\text {rel }}$. In the regions of interest for $\mathrm{d}-\alpha$ correlation function, around resonances at 42 and $84 \mathrm{MeV} / \mathrm{c}$, it is possible to obtain an improvement of efficiency of about a factor 25 using the setup with nine telescopes. A similar improvement is observed for relative energy reconstruction.
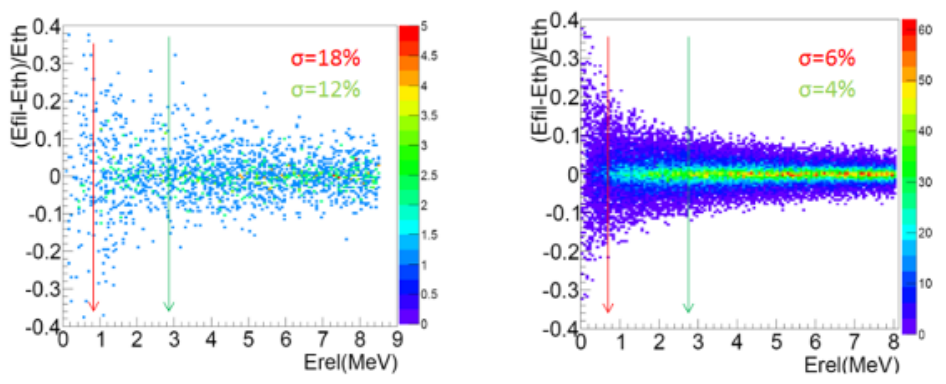

Figure 4. Deviation between the simulated relative energy and the same observable filtered by the chosen apparatus: only one telescope (left panel) and nine telescopes (right panel).

Fig. 4 shows that also in this case, in the same regions of the $d-\alpha$ correlation function the energy resolution increases by a factor around 3. So with an appropriate setup it is possible to achieve efficiency and resolution required for a proper reconstruction of the correlation functions.

\section{The importance of energy resolution}

The overall energy resolution of FARCOS telescope depends mainly on the quality of the CsI(Tl) crystals and on their light response. Several factors such as dopant concentration gradient, imperfections in crystal wrapping and/or in photodiode coupling can affect the energy resolution of $\mathrm{CsI}(\mathrm{Tl})$. In particular the aspect that deserves special attention is a possible position dependence of light output of these crystals. For this reason dedicated tests have been carried out using firstly a collimated monochromatic alpha source to scan the surface of crystal and after, different particles beams to investigate a possible non uniformity in depth, searching for notable differences in the output signal. The three-dimensional plots in fig.5 represent the comparison between surface and depth light response of one of the analyzed crystals.
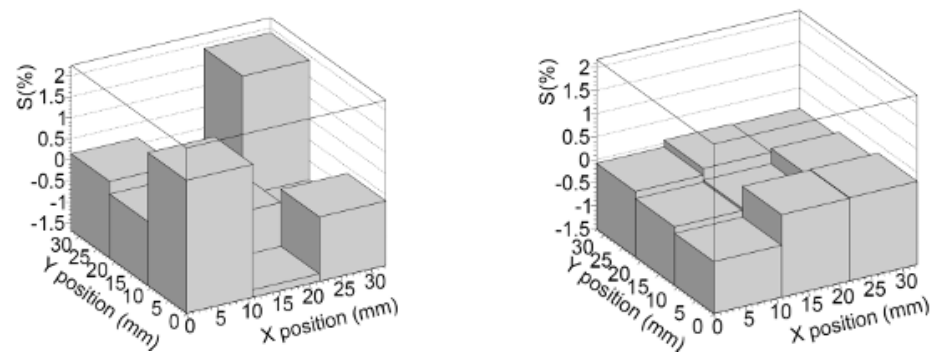

Figure 5. Characterization of light response of one of CsI(Tl) crystals obtained with alpha source (left panel) and with alpha particles beam at $62 \mathrm{AMeV}$ scattered by ${ }^{208} \mathrm{~Pb}$ (right panel) [3]. 
In particular nine different points were chosen on the surface of crystal and for each point the nonuniformity was calculated by the following formula: $S_{i j}=\left(L_{i j}-\langle L\rangle\right) /\langle L\rangle$ [4], where $L_{i j}$ represents the centroid of the energy spectrum at ij position, while $\langle L\rangle$ represents the average over the entire crystal. Comparing alpha source scanning with beam scatter (fig.5) it is possible to observe that in depth this non-uniformity is lower than on surface.
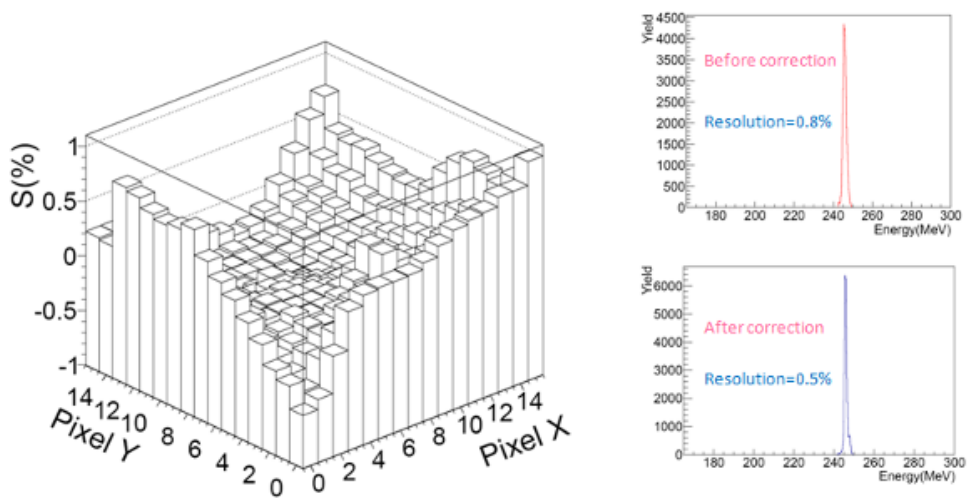

Figure 6. Left panel: Characterization of light response for one of the tested CsI(Tl) using an alpha beam at 62 $\mathrm{AMeV}$ scattered by ${ }^{208} \mathrm{~Pb}$. Right panel: total energy spectrum of $\mathrm{CsI}(\mathrm{Tl})$ crystal before and after correcting for the light response of each pixel with respect to the average response over the entire crystal.

The left panel of fig.6 reports the light response obtained performing an analysis with alpha beam 62 $\mathrm{AMeV}$ scattered by ${ }^{208} \mathrm{~Pb}$. The knowledge of the impact position (provided by the DSSSD) will allow to correct for light response non-uniformities, thus improving the final energy resolution. Hence the importance of this study. As shown in fig.6 (right panel) energy resolution of $\mathrm{CsI}(\mathrm{Tl})$ passes from $0.8 \%$ to $0.5 \%$ after taking into account the light response of each pixel with respect to the average response of the crystal.

\section{Conclusions and perspectives}

The analyzed crystals show an uniform bulk response though some of them have significant surface non-uniformities; the possible contribution of mylar non-uniformity when doing test with alpha source exploring only the surface of crystal will be verified. Studying the non-uniformity in light response it is possible to apply corrections that can improve energy resolution of crystals. For this reason it is important to continue these studies for a deeper particle penetration into the crystals by using high energy beams. In parallel to these uniformity tests, a new front end electronics optimized for the FARCOS requirement is being developed. Actually one of the main problems related to this array is the control and processing of the signals produced by each channel (132 for each telescope). During the described tests, new dedicated prototype preamplifiers (called NPA) were tested which improved the energy resolution by $30 \%$. For the future, an ASIC electronics with a digitization system is being developed, in order to obtain more compact modules and a better processing of signal produced by individual channels.

\section{References}

1. G. Verde et al., Eur. Phys. J. A30 (2006) 81

2. W.P. Tan et al., Physical Review c69, 061304(R) 2004

3. T. Minniti Ph.D. Thesis, Messina University (2013)

4. M-J. Van Goethem et al., Nucl. Instr. Meth. A 526 (2004) 455-476

5. L. Mutterer et al., Nucl. Instr. Meth. A608 (2009) 275 